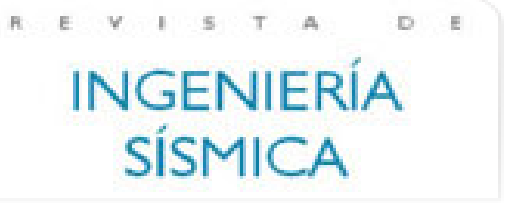

Revista de Ingeniería Sísmica

ISSN: 0185-092X

javiles@tlaloc.imta.mx

Sociedad Mexicana de Ingeniería Sísmica

México

Zárate, Gonzalo; Ayala, A. Gustavo; García, Octavio

Método sísmico estático para edificios asimétricos: revisión de enfoques

Revista de Ingeniería Sísmica, núm. 69, julio-diciembre, 2003, pp. 25-44

Sociedad Mexicana de Ingeniería Sísmica

Distrito Federal, México

Disponible en: http://www.redalyc.org/articulo.oa?id=61806902

- Cómo citar el artículo

- Número completo

- Más información del artículo

- Página de la revista en redalyc.org

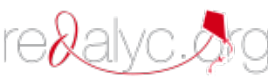

Sistema de Información Científica

Red de Revistas Científicas de América Latina, el Caribe, España y Portugal

Proyecto académico sin fines de lucro, desarrollado bajo la iniciativa de acceso abierto 


\title{
MÉTODO SÍSMICO ESTÁTICO PARA EDIFICIOS ASIMÉTRICOS: REVISIÓN DE ENFOQUES
}

\author{
Gonzalo Zárate $^{(1)}$, A. Gustavo Ayala ${ }^{(1)}$ y Octavio García ${ }^{(2)}$
}

\begin{abstract}
RESUMEN
Se revisa el método sísmico estático como una opción simplificada de análisis de estructuras asimétricas que cumplen con los requisitos reglamentarios de distribución de masas y/o rigideces. Se discuten algunas características del método como el cálculo de excentricidades estáticas y la búsqueda de la posición crítica de las cargas de diseño y como éstas características complican su uso. En este trabajo se compara un método de análisis sísmico estático propuesto por Goel y Chopra (1993), el cual consiste en la obtención del desempeño de la estructura sin el cálculo de las excentricidades de entrepiso y se compara con el método propuesto por Damy y Alcocer (1987). Se demuestra la equivalencia de resultados al usar en el análisis excentricidades de entrepiso o de nivel, aspecto importante, ya que en México se emplean exclusivamente las primeras. Se presenta una breve discusión de las ventajas y/o desventajas del método sísmico estático y del método modal espectral. Por último, se comenta la forma de aplicación de la excentricidad accidental en los métodos estático o modal espectral de acuerdo con la propuesta a las NTCDS del RCDF.
\end{abstract}

\section{SUMMARY}

The static seismic method is assessed as a simplified option for the analysis of asymmetric structures, which comply with code requirements on the distribution of masses and stiffnesses. Characteristics of the method such as the determination of the static eccentricities and the search for the critical position of the design forces and how these characteristics make the method difficult to use, are discussed. A version of the static seismic analysis method proposed by Goel and Chopra (1993), which consists of obtaining the performance of the structure without computing the eccentricities of the interstorey, is compared with the method proposed by Damy and Alcocer (1987). The equivalence of results using inter-storey eccentricities and those level eccentricities is demonstrated. This is an important fact as the generality programs available in Mexico do not have implemented the procedures needed to automatically apply the method as suggested in the code. To provide the structural engineer with a wider perspective when considering existing methods of analysis, a brief discussion is presented on the advantages and/or disadvantages in using the static seismic method or the modal spectral method. Consideration of the accidental eccentricity in the static or modal spectral methods is commented on in accordance with the Proposal to the NTCDSRCDF.

Artículo recibido el 3 de marzo de 2003 y aprobado para su publicación el 17 de junio de 2003. Se aceptarán comentarios y/o discusiones hasta cinco meses después de su publicación.

(1) Instituto de Ingeniería, UNAM, APDO 70.642 Ciudad Universitaria, 04510 México, D.F., dgzs_1999@yahoo.com, gayala@dali.fi-p.unam.mx,

${ }^{(2)}$ Facultad de Ingeniería, UNAM, Ciudad Universitaria, 04510 México, D.F. , octavio@dali.fi-p.unam.mx 


\section{INTRODUCCIÓN}

El método sísmico estático (ME) es una alternativa simplificada para el análisis de edificios que se ajustan a determinadas hipótesis de comportamiento y tipos de estructuración, cuya altura no rebasa los $60 \mathrm{~m}$ según las Normas Técnicas Complementarias para Diseño por Sismo (NTCDS) del Reglamento de Construcciones del Distrito Federal (RCDF,1993) vigente. En la actualidad se encuentra en proceso de aprobación una nueva versión de éstas normas (NTCDS, 2001) que limita aún más la altura de los edificios y considera que ésta no debe exceder $30 \mathrm{~m}$ para estructuras regulares y $20 \mathrm{~m}$ para estructuras irregulares.

Para considerar los efectos de torsión en el análisis estático, se toma en cuenta la amplificación dinámica de la torsión mediante un factor que afecta a la excentricidad estructural $\left(\mathrm{e}_{\mathrm{s}}\right)$. Además, se incluyen las incertidumbres existentes en el cálculo de las masas, rigideces, resistencias de elementos y otras fuentes de torsión no consideradas en el análisis, mediante una excentricidad accidental expresada como una fracción de la longitud de la planta ortogonal a la dirección considerada de análisis (b). Estos dos tipos de excentricidades definen la llamada excentricidad de diseño por torsión, utilizada en México y la mayoría de los códigos de otros países; ésta excentricidad se expresa de la forma: $e_{\mathrm{s} 1}=\alpha e_{\mathrm{s}}+\beta b$ y $e_{\mathrm{s} 2}=\delta e_{\mathrm{s}}-\beta b$. Para el caso del $\mathrm{RCDF}$ los factores involucrados en la definición de la excentricidad de diseño valen $\alpha=1.5, \delta=1.0$ y $\beta=0.1$.

En el ME, la determinación de la excentricidad estructural requiere del cálculo de las coordenadas del centro de rigidez, sin embargo, resulta complicado establecerlas para un edificio de varios niveles ya que los programas comerciales existentes, generalmente no tienen implementados los procedimientos y formulaciones matemáticas, o bien como se comenta en (Goel y Chopra, 1993), existen otros métodos simplificados que requieren de modelos equivalentes que representan a la estructura.

Por otro lado, a diferencia de lo que ocurre en edificios de un nivel, para los edificios de varios niveles existen diferentes definiciones de los centros de rigidez. En la tabla 1 se presentan algunas de estas definiciones y/o los métodos que se derivan de ellas. Cabe aclarar que en México para la aplicación del ME, se usa el concepto de centro de torsión, que es el punto en el entrepiso en el cual actúa el cortante sísmico para que únicamente exista traslación.

En México para la aplicación del ME según la normatividad vigente y las (NTCDS, 2001), es necesario el cálculo de la excentricidad estructural de entrepiso. Esto a pesar de que en el trabajo de (Bazán, 1978), se formula un método matricial que permite aplicar el ME de acuerdo al RCDF sin necesidad de encontrar la posición de los centros de torsión. En la misma línea que el reglamento, (Damy y Alcocer, 1987) proponen una metodología de cálculo que permite obtener las coordenadas de los centros de torsión (en adelante Método de Damy). Con fines ilustrativos, en la fig. 1 se muestran las distintas coordenadas a las que se hace referencia en el RCDF y en este trabajo.

En un trabajo conceptualmente relacionado con el realizado por Bazán (Goel y Chopra,1993), usan la definición de centros de rigidez propuesta por (Cheung y Tso, 1986a, 
1986b) para formular un procedimiento que, evitando la determinación de las coordenadas de los centros de rigidez de nivel, permite obtener la respuesta del edificio como si se hubiera aplicado el ME de manera convencional de acuerdo al (UBC,1991). En adelante este método se denominará Método de Goel. La ventaja de éste método, respecto al de Damy, reside en que el post-procesamiento requerido por los resultados de un programa convencional de análisis de edificios es mínimo, puesto que únicamente hay que combinar linealmente los resultados de tres análisis con modificaciones menores a un modelo estructural.

Tabla 1. Centros de rigidez. Definiciones y métodos

\begin{tabular}{|c|c|l|}
\hline AÑO & AUTOR(ES) & \multicolumn{1}{c|}{ DEFINICIÓN / MÉTODO PROPUESTO } \\
\hline 1977 & Poole & $\begin{array}{l}\text { Define el centro de rigidez de un entrepiso como la posición de la } \\
\text { resultante de fuerzas cortantes de varios elementos resistentes en ese } \\
\text { entrepiso, cuando el edificio se somete a cargas laterales estáticas que } \\
\text { no causan rotación en ninguno de sus niveles. }\end{array}$ \\
\hline 1978 & Bazán & $\begin{array}{l}\text { Propone un método que permite determinar las acciones de diseño en un } \\
\text { edificio de acuerdo al Reglamento de Construcciones del DF, 1976. El } \\
\text { método usa implícitamente el concepto de centro de torsión de nivel. }\end{array}$ \\
\hline 1984 & Humar & $\begin{array}{l}\text { Define el centro de rigidez en cualquier nivel como el punto en el que la } \\
\text { aplicación de una carga lateral no causa rotación en ese nivel, sin } \\
\text { embargo, los otros niveles pueden rotar. }\end{array}$ \\
\hline 1986 & Cheung y Tso & $\begin{array}{l}\text { Definen al centro de rigidez para edificios con marcos ortogonales como } \\
\text { el conjunto de puntos localizados en sus niveles en los cuales, la } \\
\text { aplicación de las cargas laterales no causa rotación de ningún nivel. }\end{array}$ \\
\hline 1987 & $\begin{array}{l}\text { Presentan una metodología para obtener el centro de torsión de entrepiso } \\
\text { a partir de la matriz de rigideces de un edificio, definiendo al centro de } \\
\text { torsión como el punto por el que debe pasar la línea de acción de la } \\
\text { fuerza cortante sísmica para que el movimiento relativo de los niveles } \\
\text { que limitan el entrepiso sea exclusivamente de traslación. }\end{array}$ \\
\hline
\end{tabular}

En apariencia, el método de Goel y el método de Damy no son equivalentes, debido a que el Reglamento de Construcciones del Distrito Federal (RCDF) requiere utilizar excentricidades de entrepiso (aspecto que considera el método de Damy), mientras que el método de Goel utiliza excentricidades de nivel en consistencia con el reglamento americano. Sin embargo, como se demuestra mas adelante, ambos métodos son equivalentes (Zárate, 2002).

Con el fin de comparar el Método de Damy y el Método de Goel desde el punto de vista de igualdad de resultados así como de facilidad de uso, en éste artículo se presentan los procedimientos para su aplicación en cualquier programa de análisis estructural que permita modelar edificios con diafragmas de piso rígidos. Para fines ilustrativos, se muestra, para cada método, el procedimiento considerando solo una dirección de análisis.

\section{MÉTODO DE DAMY}

(Damy y Alcocer,1987) presentan un procedimiento que permite obtener los centros de torsión de todos los entrepisos a partir de la matriz de rigidez de un edificio de varios niveles, con objeto de 
determinar la excentricidad estática y aplicar el ME de acuerdo con el RCDF. La metodología presentada considera que el edificio está formado por marcos planos y/o muros ligados entre si por sistemas de piso que se consideran como diafragmas indeformables en su plano (diafragmas rígidos). Es de interés mencionar que la mayoría de los programas comerciales disponibles en México no tienen implementado tal procedimiento por lo que, su empleo, requiere del postprocesamiento de una gran cantidad de información, usando herramientas distintas al programa con el que se analizó la estructura.
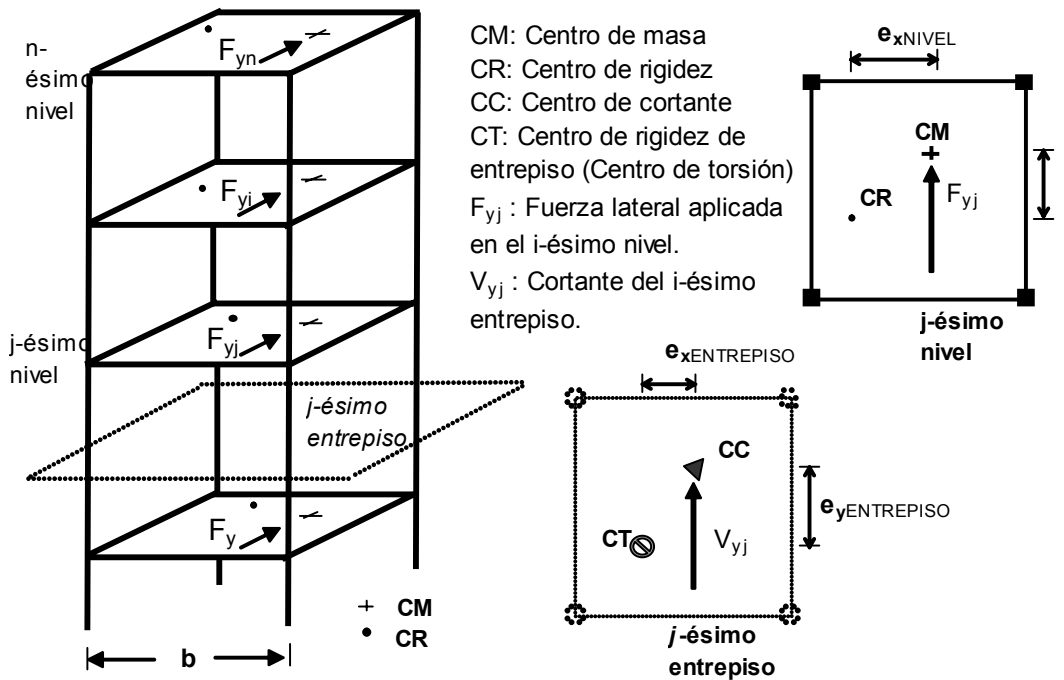

Figura 1. Coordenadas de referencia para la aplicación del ME.

A continuación se presenta la versión del llamado método de Damy empleando cualquier programa de análisis estructural con las características mencionadas; para ilustrar ésta metodología se seleccionó un edificio con marcos ortogonales y las fuerzas sísmicas actuando en dirección "Y", de acuerdo al sistema de referencia indicado. Los pasos a seguir son:

1. Análisis de cargas laterales en la dirección de interés, considerando que los niveles no experimentan rotación. Para lograr esto, la fuerza sísmica debe pasar por el centro de torsión, como se indica en la fig 2. Como los marcos son ortogonales, existirá desplazamiento únicamente en la dirección de carga.

2. Se calculan las coordenadas de los centros de torsión de entrepiso $\left(\mathrm{X}_{\mathrm{CRj}}\right)$, a partir de los momentos de entrepiso $\left(\mathrm{M}_{\mathrm{sj}}\right)$ generados por los cortantes en cada columna, fig. 3, debidos al paso 1 , respecto a un eje vertical, ec. 1 .

$X_{C R j}=\frac{M_{s j}}{V_{j}}$ 


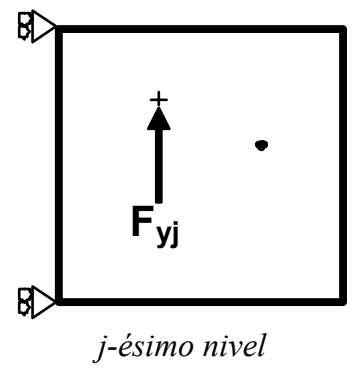

Figura 2. Análisis de traslación

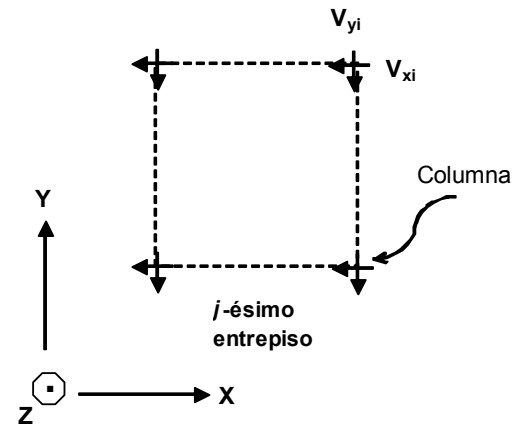

Figura 3. Cálculo de los momentos de entrepiso

3. Determinación de las coordenadas de los centros de cortante $\left(\mathrm{X}_{\mathrm{CC}}\right)$ de los entrepisos a partir de las coordenadas de los centros de masas $\left(\mathrm{X}_{\mathrm{Mj}}\right)$ de los niveles, ec. 2.

$X_{C C j}=\sum_{j}^{N} F_{j} X_{C M j} / V_{j}$

4. Determinación de las excentricidades estáticas de entrepiso, ec 3.

$e_{s x j}=X_{C R j}-X_{C C j}$

5. Obtención de las excentricidades de diseño, en el entrepiso, dadas por las ecs. 4 y 5.

$e_{d x j}=1.5 e_{s x j}+0.1 b$

$e_{d x j}=e_{s x j}-0.1 b$

6. Cálculo de los momentos de entrepiso modificados $\left(\mathrm{M}_{\mathrm{dj}}\right)$ generados por la nueva posición de la fuerza cortante a partir de las excentricidades de diseño.

7. Cálculo de los momentos de nivel a partir de la resta de los momentos de dos entrepisos sucesivos y determinación de las nuevas coordenadas de los puntos de aplicación de las fuerzas laterales $\left(\mathrm{X}_{\mathrm{dj}}\right)$ :

$X_{d j}=\frac{M_{d j}-M_{d j+1}}{F_{Y j}}$

8. Análisis tridimensional de la estructura, considerando las posibles combinaciones de carga para el diseño de la misma. 


\section{MÉTODO DE GOEL}

Con objeto de simplificar la determinación de las fuerzas de diseño obtenidas con el ME, (Goel y Chopra, 1993) presentan un procedimiento que permite obtener la respuesta de una estructura analizada usando el ME sin necesidad de determinar los centros de rigidez de nivel. El método propuesto conduce a resultados idénticos a los que se obtienen cuando se aplica esta versión del ME en su forma convencional; el resultado final del análisis se obtiene al combinar linealmente las respuestas derivadas de tres análisis estáticos para tres condiciones de carga aplicadas en los centros de masas.

De igual forma que en el método de Damy, para cada análisis todos los niveles tendrán simultáneamente las mismas condiciones de restricción y carga, mostrándose, para fines ilustrativos, únicamente un nivel cualquiera de un edificio. Los pasos a seguir son:

1. Aplicación de las fuerzas laterales en los centros de masas de cada nivel, permitiendo únicamente traslación en la dirección de carga $\left(\mathrm{r}^{(1)}\right)$; para fines de comportamiento, si se evita la rotación, la fuerza sísmica puede aplicarse en el centro de masa o en el centro de rigidez como en el caso de la fig. 2.

2. Aplicación de cargas laterales en los centros de masas de cada nivel, permitiendo traslación y rotación de los niveles, $\left(\mathrm{r}^{(2)}\right)$, fig. 4.

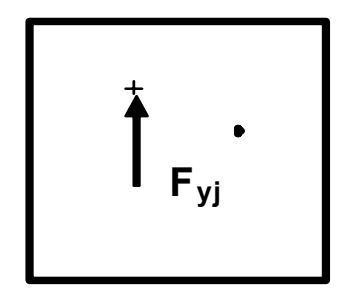

Figura 4. Análisis con traslación y rotación de los niveles

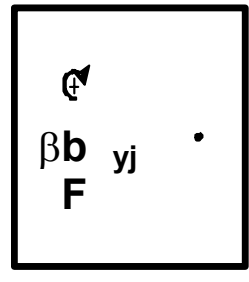

Figura 5. Momento debido a la excentricidad accidental

3. Aplicación en el nivel (por ejemplo, centro de masas) de un momento debido a la excentricidad accidental $\left(\mathrm{r}^{(3)}\right)$, fig. 5.

4. Combinación de las respuestas obtenidas en los pasos 1,2 y $3\left(r^{(1)}, r^{(2)}, r^{(3)}\right.$ respectivamente) de acuerdo con la ec. 7.

$r=r^{(1)}+\gamma\left(r^{(2)}-r^{(1)}\right) \pm r^{(3)}$

donde $\gamma$ es el coeficiente de amplificación/deamplificación dinámica.

$r^{(a)}=r^{(1)}+1.5\left(r^{(2)}-r^{(1)}\right)+r^{(3)}$ 
$r^{(b)}=r^{(1)}+\left(r^{(2)}-r^{(1)}\right)-r^{(3)}$

Es importante mencionar que es necesario ser cuidadoso para obtener la respuesta deseada al considerar el signo correcto de $\mathrm{r}^{(3)}$ (i.e. la respuesta debida a la excentricidad accidental). Para tal fin y de acuerdo con (Goel y Chopra, 1995) se procede de la siguiente manera:

Si $r^{(1)}$ es menor que $\mathrm{r}^{(2)}$, el signo de $\mathrm{r}^{(3)}$ deberá ser tal que incremente la suma de los primeros dos términos en $\mathrm{r}^{(\mathrm{a})}$, ec. 8 , y que reduzca la suma de los primeros dos términos en la ecuación $\mathrm{r}^{(\mathrm{b})}$, ec. 9. Por otro lado, si $\mathrm{r}^{(1)}$ es más grande que $\mathrm{r}^{(2)}$, el signo de $\mathrm{r}^{(3)}$ deberá tomarse tal que incremente la suma de los dos primeros términos en $\mathrm{r}^{(\mathrm{b})}$, ec. 9 , y que reduzca $\mathrm{r}^{(\mathrm{a})}$, ec. 8 .

5. La respuesta de diseño será la mayor de entre $\mathrm{r}^{(\mathrm{a})} \mathrm{y}^{(\mathrm{b})}$. Por considerarlo de interés, en el Apéndice I se presenta una demostración de la propuesta de Goel y Chopra.

\section{DEMOSTRACIÓN DE LA EQUIVALENCIA DE MÉTODOS}

Sea el i-ésimo nivel y el i-ésimo entrepiso de un edificio como el que se muestra en la fig. 6.
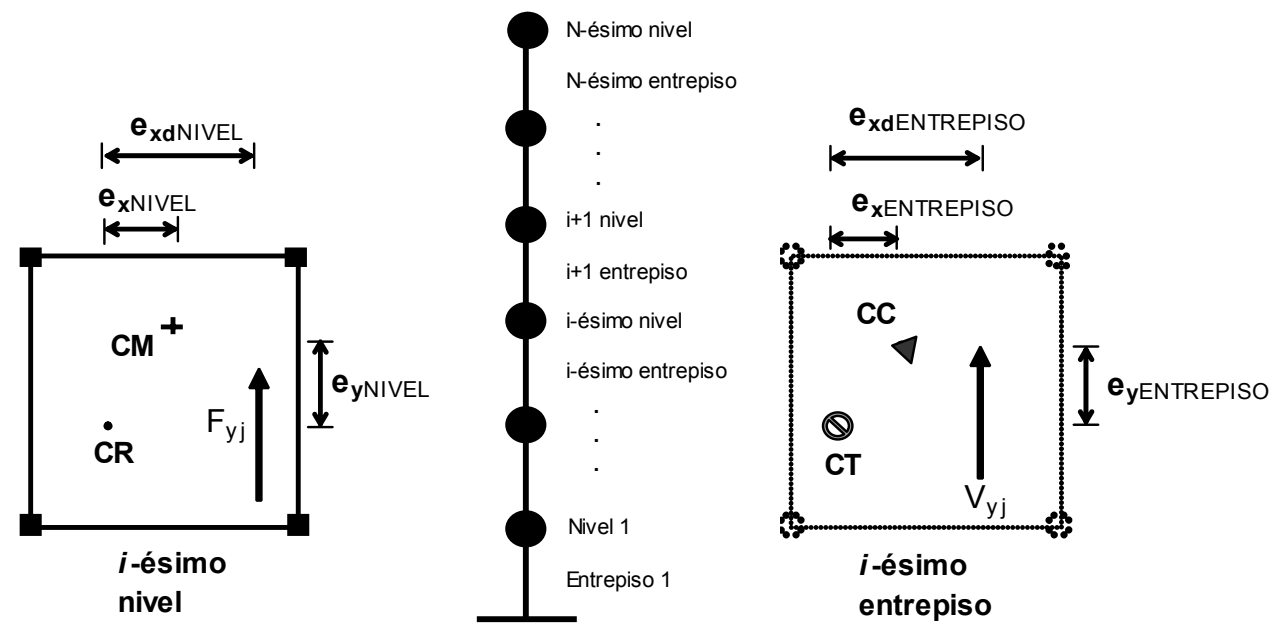

Figura 6. Edificio de varios niveles. Definición de excentricidades de nivel y entrepiso.

La excentricidad de diseño para el i-ésimo nivel $\left(\mathrm{e}_{\mathrm{dNi}}\right)$ o el i-ésimo entrepiso $\left(\mathrm{e}_{\mathrm{dEi}}\right)$ en una dirección de análisis, fig. 6, se expresa mediante la ec. 10; en ésta E denota entrepiso y N, nivel; $\gamma$ toma los valores de 1.0 o 1.5 según sea el caso, considerándose el signo correspondiente para el término de la excentricidad accidental $( \pm \beta b)$. Es claro que la excentricidad de diseño $e_{\text {dai }}$ puede expresarse como la suma de la excentricidad estática $\left(\mathrm{e}_{\mathrm{s \alpha i}}\right)$ más un incremento debido a la amplificación/deamplificación dinámica y excentricidad accidental $\left(\mathrm{e}_{\alpha \mathrm{i}}\right)$, fig. 7 . En adelante $\mathrm{e}_{\alpha \mathrm{i}}$ será llamada excentricidad adicional, ec. 11.

$e_{d \alpha i}=\gamma e_{s \alpha i} \pm \beta b, \quad \alpha=E, N$ 


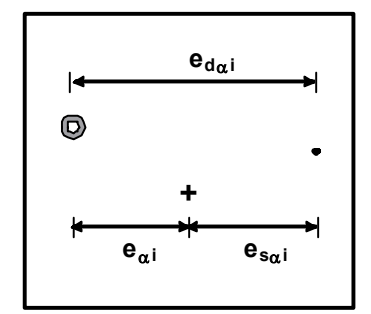

Posición de diseño de la fuerza

Figura 7. Excentricidad de diseño (i-ésimo nivel o entrepiso).

$e_{\alpha i}=\Delta e_{s \alpha i} \pm \beta b, \quad \Delta=\gamma-1$

Los momentos de dos entrepisos sucesivos debidos al cortante sísmico para la dirección considerada $\left(\mathrm{V}_{\mathrm{Yi}}\right)$, se obtienen con las ecs. 12 y 13.

$M s_{i+1}=V_{Y i+1} X_{C C i+1}$

$M s_{i}=V_{Y i} X_{C C i}$

La coordenada $\mathrm{X}_{\mathrm{CCi}}$ del centro de cortante para el i-ésimo entrepiso se obtiene con la ec. 14 , donde, $\mathrm{X}_{\mathrm{CMi}}$ es la coordenada del centro de masas de cada nivel en la dirección perpendicular a la dirección analizada. Los momentos de diseño asociados a las ecs. 12 y 13 al considerar la excentricidad adicional correspondiente $\left(\mathrm{e}_{\mathrm{Ei}}\right)$ se calculan con las ecs. 15 y 16.

$X_{C C i}=\frac{\sum_{i}^{N} F_{Y i} X_{C M i}}{V_{Y i}}$

$M d_{i+1}=V_{Y i+1}\left(X_{C C i+1}+e_{E i+1}\right)$

$M d_{i}=V_{Y i}\left(X_{C C i}+e_{E i}\right)$

donde:

$V_{Y i}=\sum_{i}^{N} F_{Y i} \Rightarrow V_{Y i}=F_{Y i}+\sum_{i+1}^{N} F_{Y i}$

Restando los momentos de diseño de entrepisos sucesivos, se tiene la ec. 18

$M d_{i}-M d_{i+1}=V_{Y i}\left(X_{C C i}+e_{E i}\right)-V_{Y i+1}\left(X_{C C i+1}+e_{E i+1}\right)$

Al sustituir las ecs. 14 a 17, la ec. 18 queda expresada como se indica la ec. 19. 
$M d_{i}-M d_{i+1}=\left(F_{Y i}+\sum_{i+1}^{N} F_{Y i}\right)\left\{\frac{\sum_{i+1}^{N} F_{Y i} X_{M i}+F_{Y i} X_{C M i}}{V_{Y i+1}+F_{Y i}}+e_{E i}\right\}-\sum_{i+1}^{N} F_{Y i}\left\{\frac{\sum_{i+1}^{N} F_{Y i} X_{C M i}}{V_{Y i+1}}+e_{E i+1}\right\}$

que conduce a la ec. 20 .

$M d_{i}-M d_{i+1}=F_{Y i} X_{C M i}+e_{E i} V_{Y i}-e_{E i+1} V_{Y i+1}$

Sustituyendo la ec. 11 en la ec. 20 y asociando términos, resulta la ec. 21.

$M d_{i}-M d_{i+1}=\Delta\left(e_{S E i} V_{Y i}-e_{S E i+1} V_{Y i+1}\right) \pm \beta b\left(V_{Y i}-V_{Y i+1}\right)+F_{Y i} X_{C M i}$

Por otro lado, el momento de diseño para el i-ésimo nivel considerando la excentricidad adicional en el entrepiso $\left(\mathrm{e}_{\mathrm{Ni}}\right)$ se obtiene con la ec.22.

$F_{Y i}\left(X_{C M i}+e_{N i}\right)=X_{C M i} F_{Y i}+\Delta e_{S N i} F_{Y i} \pm \beta b F_{Y i}$

Se busca demostrar que el uso de excentricidades de nivel o de entrepiso conduce a los mismos resultados, por tanto al igualar las ecs. (21) y (22) se obtiene la ec. 23.

$\Delta\left(e_{s E i} V_{i}-e_{s E i+1} V_{i+1}\right) \pm \beta b F_{i}+F_{i} X_{C M i}=F_{Y i} X_{C M i}+\Delta_{Y i} e_{s N i} F_{Y i} \pm \beta b F_{Y i}$

que se reduce a la expresión indicada en la ec. 24 .

$e_{s N i} F_{i}=e_{s E i} V_{i}-e_{s E i+1} V_{i+1}$

Por definición, la excentricidad estática de entrepiso, se evalúa con la ec.25.

$e_{S E i}=X_{C C i}-X_{C T i}=\frac{\sum_{i}^{N} F_{Y i} X_{C M i}}{V_{Y i}}-\frac{M_{E i}}{V_{Y i}}$

donde $\mathrm{M}_{\mathrm{Ei}}$ es el momento de entrepiso debido a la aplicación del cortante sísmico en el centro de rigidez correspondiente. Sustituyendo la ec. 25 en la ec. 24, se deriva la ec. 26.

$V_{Y i} e_{S E i}-V_{Y i+1} e_{S E i+1}=F_{Y i} X_{C M i}+M_{E i+1}-M_{E i}$

$\mathrm{M}_{\mathrm{Ei}+1}$ y $\mathrm{M}_{\mathrm{Ei}}$ son los momentos de entrepisos sucesivos necesarios para que éstos no roten. Al ser el desplazamiento angular de los entrepisos nulo, también será nulo el desplazamiento angular del nivel que se encuentra entre ellos, lo cual es equivalente a decir que la fuerza sísmica 
está aplicada en el centro de rigidez de nivel, lo anterior se considera en toda la altura del edificio. De ésta manera el momento en nivel se obtiene con la ec 27.

$V_{Y i} e_{s E i}-V_{Y i+1} e_{s E i+1}=F_{Y i} X_{C M i}-F_{Y i} X_{C R}$

Asociando términos, la ec. 27 se puede presentar como se indica en la ec. 28.

$V_{Y i} e_{S E i}-V_{Y i+1} e_{S E i+1}=F_{Y i}\left(X_{C M i}-X_{C R}\right)=e_{S N i} F_{Y i}$

Al comparar la ec. 28 con la ec. 24 se verifica la igualdad propuesta. Con esto se demuestra que para la dirección analizada, la consideración de excentricidad de nivel o de entrepiso conduce a iguales resultados. Para la dirección ortogonal se procede de manera análoga.

Es importante comentar un par de aspectos que son de interés en esta demostración, ambos relacionados con los requisitos de aplicación del ME. El primero de ellos corresponde a la fracción de la planta ortogonal a la dirección de análisis que se considera para determinar la contribución de la excentricidad accidental $(\beta b)$. El término que corresponde a esta excentricidad únicamente puede expresarse como se presenta en la ec. 21 si su magnitud se considera constante en la altura del edificio, aspecto que no cumplen todos los edificios susceptibles de ser analizados con el ME, se recomienda considerar la dimensión b como la que resulta del promedio de las dimensiones de todos los niveles del edificio debido a que no existe una justificación formal para considerar una fracción de la longitud de la planta del edificio asociándola a una excentricidad accidental, pues a todas luces b es una propiedad geométrica del edificio y nada tiene que ver con una posible excentricidad accidental, además de que dicha excentricidad se aplica en cualquier modalidad de análisis que se adopte.

Por otro lado, si bien es cierto que el Método de Damy es de carácter general por haber sido desarrollado para el análisis de estructuras constituidas por planos resistentes orientados arbitrariamente, el RCDF permite el uso del ME exclusivamente en estructuras constituidas por marcos ortogonales o sensiblemente ortogonales, siendo en este aspecto por completo equivalente al Método de Goel que considera a las estructuras formadas únicamente por marcos ortogonales.

Es importante mencionar que, en su aplicación, las normas de diseño requieren que se tomen cuenta los efectos bidireccionales de la acción sísmica mediante la aplicación de fuerzas estáticas laterales en los niveles del edificio en direcciones ortogonales.

\section{EJEMPLO DE APLICACIÓN}

\section{Descripción del edificio}

Para ilustrar la equivalencia de los métodos propuestos por Damy y por Goel, se considera un edificio cuya dirección de análisis, configuración en planta y elevación así como las demás propiedades que lo definen se muestran en la fig. 8; las fuerzas laterales se calculan usando un 
coeficiente sísmico (c) de 0.4 y un factor de comportamiento sísmico (Q) de 4, sus magnitudes se presentan en la tabla 2. Para fines prácticos sólo se utiliza una de las excentricidades de diseño, ec.4, establecida por las (NTCDS, 1995).

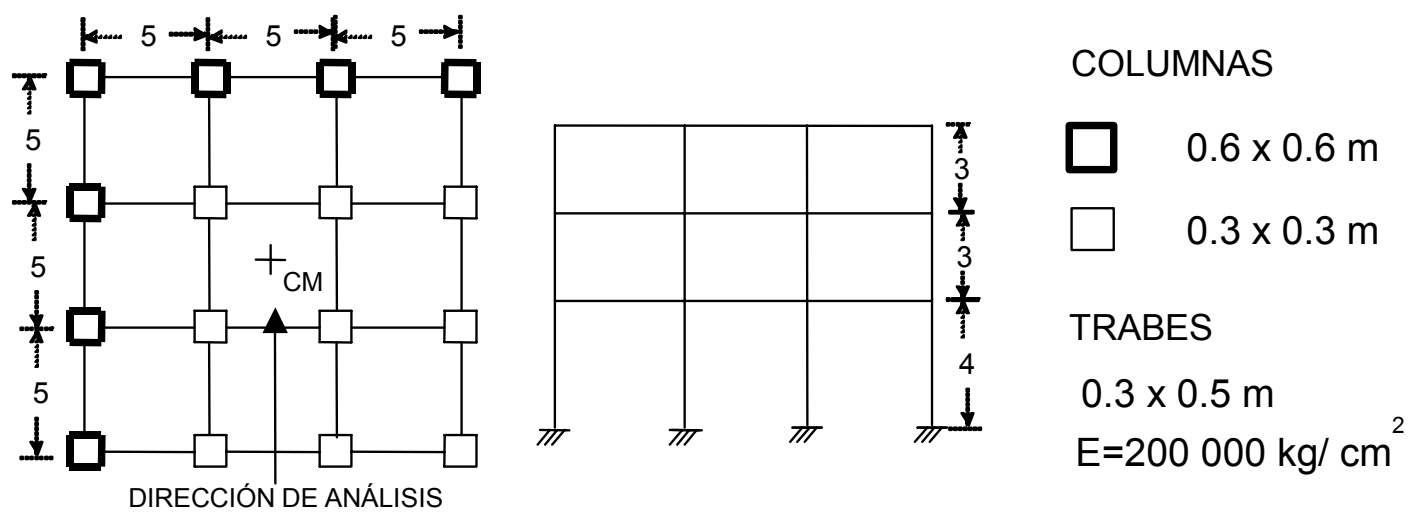

Figura 8. Características del edificio analizado

\section{Aplicación del método de Damy}

Para los fines comparativos, se denominó Procedimiento I al Método de Damy considerando el cálculo de la excentricidad de diseño en el entrepiso. Por otro lado, se utilizará un Procedimiento II que considera el cálculo de la excentricidad de diseño en el nivel. Cabe aclarar que el Procedimiento II es una modificación del Procedimiento I, pues únicamente usa los centros de rigidez de nivel, los cuales pueden obtenerse matricialmente, (Chipol, 2001), o bien usando parte de la metodología descrita en este artículo. En ambos casos los resultados a comparar serán las coordenadas finales de los puntos de aplicación de las fuerzas laterales, presentadas en la tabla 2. En las tablas 3, 4 y 5 se resume la secuela de cálculo de las coordenadas finales de aplicación de dichas fuerzas.

Tabla 2. Distribución de fuerzas laterales.

\begin{tabular}{|c|c|c|c|c|c|}
\hline NIVEL & $\mathrm{W}_{\mathrm{i}}$ (Ton) & $\mathrm{h}_{\mathrm{i}}(\mathrm{m})$ & $\mathrm{W}_{\mathrm{i}}$ hi (Ton $\left.\mathrm{m}\right)$ & $\mathrm{F}_{\mathrm{i}}$ (Ton) & $\mathrm{V}_{\mathrm{i}}$ (Ton) \\
\hline 3 & 90 & 10 & 900 & 13.30 & 13.30 \\
\hline 2 & 114.75 & 7 & 803.25 & 11.87 & 25.17 \\
\hline 1 & 114.75 & 4 & 459 & 6.78 & 31.95 \\
\hline$\Sigma$ & 319.5 & & 2162.25 & & \\
\hline
\end{tabular}

Procedimiento I. Consideración de la excentricidad estática en el entrepiso. La excentricidad estática se calcula como la distancia entre el centro de cortante y el centro de torsión ó centro de rigidez de entrepiso, (NTCDS, 1995) y (NTCDS, 2001). 
Gonzalo Zárate, A. Gustavo Ayala y Octavio García

Tabla 3. Determinación de momentos de entrepiso.

\begin{tabular}{|c|c|c|c|c|c|c|c|}
\hline ENTREPISO & $\mathrm{Xcc}(\mathrm{m})$ & $\mathrm{Xct}(\mathrm{m})$ & $\mathrm{e}_{\mathrm{sx}}(\mathrm{m})$ & $\mathrm{Ed}_{1 \mathrm{x}}(\mathrm{m})$ & $\mathrm{Xcc}_{1}(\mathrm{~m})$ & $\mathrm{V}_{\text {sIsmico }}($ ton $)$ & $\mathrm{M}_{\mathrm{d}}($ ton-m) \\
\hline 3 & 7.500 & 5.471 & 2.029 & 4.544 & 10.015 & 13.300 & 60.429 \\
\hline 2 & 7.500 & 5.395 & 2.105 & 4.658 & 10.053 & 25.170 & 117.229 \\
\hline 1 & 7.500 & 4.831 & 2.669 & 5.504 & 10.335 & 31.950 & 175.837 \\
\hline
\end{tabular}

donde $\mathrm{e}_{\mathrm{d} 1 \mathrm{x}}$ :es la excentricidad de diseño y $\mathrm{X}_{\mathrm{cc} 1}$ la nueva posición de la fuerza cortante en el entrepiso. $\mathrm{X}_{\mathrm{ccl}}=\mathrm{X}_{\mathrm{ct}}+\mathrm{ed}_{1 \mathrm{x}}$

Tabla 4. Determinación de coordenadas de aplicación de fuerzas sísmicas por el Procedimiento I

\begin{tabular}{|c|c|c|c|c|c|c|c|}
\hline ENTREPISO & $\mathrm{M}($ ton $\mathrm{m})$ & NIVEL & $\mathrm{M}_{\mathrm{dj}}(\mathrm{ton} \mathrm{m})$ & $\mathrm{F}_{\mathrm{j}}(\mathrm{m})$ & $\mathrm{ed}_{\mathrm{x}}(\mathrm{m})$ & $\mathrm{X}_{\mathrm{CR}}(\mathrm{m})$ & $\mathrm{X}_{\mathrm{d}}(\mathrm{m})$ \\
\hline & & 3 & 60.429 & 13.300 & 4.544 & 5.471 & 10.015 \\
\hline 3 & 60.429 & & & & & & \\
\hline 2 & 117.229 & & & & & & \\
\hline & & 1 & 58.608 & 6.780 & 8.644 & 2.734 & 11.378 \\
\hline 1 & 175.837 & & & & & & \\
\hline
\end{tabular}

Procedimiento II. Consideración de la excentricidad estática en el nivel. La excentricidad estática se calcula como la distancia entre el centro de masas y el centro de rigidez de nivel.

Tabla 5. Determinación de coordenadas de aplicación de fuerzas sísmicas usando el Procedimiento II.

\begin{tabular}{|c|c|c|c|c|c|}
\hline NIVEL & $\mathrm{X}_{\mathrm{CM}}(\mathrm{m})$ & $\mathrm{X}_{\mathrm{ct}}(\mathrm{m})$ & $\mathrm{e}_{\mathrm{sx}}(\mathrm{m})$ & $\mathrm{e}_{\mathrm{dx}}(\mathrm{m})$ & $\mathrm{X}_{\mathrm{d}}(\mathrm{m})$ \\
\hline 3 & 7.500 & 5.471 & 2.029 & 4.543 & 10.014 \\
\hline 2 & 7.500 & 5.311 & 2.189 & 4.784 & 10.095 \\
\hline 1 & 7.500 & 2.734 & 4.766 & 8.649 & 11.383 \\
\hline
\end{tabular}

En la tabla $5 \mathrm{X}_{\mathrm{CM}}$ es la coordenada del centro de masas en la dirección de análisis.

\section{Aplicación del método de Goel}

El método de Goel, al igual que el de Damy, requiere del post-procesamiento de resultados derivados del análisis base. Para la determinación de los cortantes basales de cada marco, únicamente se necesitan los cortantes asociados a las columnas del primer entrepiso. La definición de los marcos para el edificio estudiado se muestra en la fig. 9 mientras que los cortantes basales que se obtienen como respuesta de las etapas de análisis propuestas por el método se ilustran en las tablas 6 y 7 . La respuesta total de la estructura se indica en la tabla 8. 
La respuesta de la estructura analizada se obtiene combinando las respuestas de los análisis presentados en las tablas 6 y 7 con la ec. 8. Los cortantes basales que se obtienen se presentan en la tabla 8 .

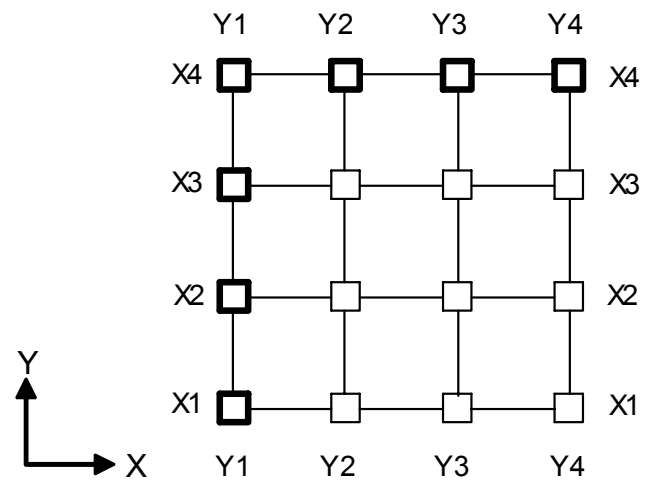

Figura 9. Definición de marcos para el edificio.

Tabla 6. Cortantes basales para marcos paralelos a $X$ debidos a las condiciones $\mathbf{r}^{(1)}, \mathbf{r}^{(2)}, \mathbf{r}^{(3)}$.

\begin{tabular}{|c|c|c|c|}
\hline MARCO & $\mathbf{r}^{(\mathbf{1})}$ & $\mathbf{r}^{(2)}$ & $\mathbf{r}^{(\mathbf{3})}$ \\
\hline $\mathrm{X} 1-\mathrm{X} 1$ & -0.002 & -2.114 & -1.189 \\
\hline $\mathrm{X} 2-\mathrm{X} 2$ & 0.003 & -1.07 & -0.603 \\
\hline $\mathrm{X} 3-\mathrm{X} 3$ & -0.008 & -0.044 & -0.02 \\
\hline $\mathrm{X} 4-\mathrm{X} 4$ & -0.004 & 3.228 & 1.812 \\
\hline$\Sigma$ & $\mathbf{- 0 . 0 1 1}$ & $\mathbf{0}$ & $\mathbf{0}$ \\
\hline
\end{tabular}

Tabla 7. Cortantes basales para marcos paralelos a $Y$ debidos a las condiciones $\mathbf{r}^{(1)}, \mathbf{r}^{(2)}, \mathbf{r}^{(3)}$.

\begin{tabular}{|c|c|c|c|}
\hline MARCO & $\mathbf{r}^{(\mathbf{1})}$ & $\mathbf{r}^{(2)}$ & $\mathbf{r}^{(3)}$ \\
\hline Y1-Y1 & -16.516 & -13.289 & 1.812 \\
\hline Y2-Y2 & -5.145 & -5.183 & -0.02 \\
\hline Y3-Y3 & -5.144 & -6.219 & -0.603 \\
\hline Y4-Y4 & -5.145 & -7.259 & -1.189 \\
\hline$\Sigma$ & $\mathbf{- 3 1 . 9 5}$ & $\mathbf{- 3 1 . 9 5}$ & $\mathbf{0}$ \\
\hline
\end{tabular}

\section{Comparación de resultados}

En este apartado se muestran los resultados que se obtienen de la aplicación del Método de Goel y del Método de Damy. La tabla 9 muestra las coordenadas finales de aplicación de las fuerzas laterales derivadas de la aplicación del Método de Damy en las modalidades de entrepiso y de nivel (Procedimientos I y II respectivamente). Las diferencias que se presentan se deben a problemas de precisión asociados al procesamiento de los datos. 
Tabla 8. Respuesta total de la estructura usando el Método de Goel.

\begin{tabular}{|c|c|c|c|c|}
\hline MARCO & CORTANTE BASAL (ton) & & MARCO & CORTANTE BASAL (ton) \\
\hline $\mathrm{X} 1-\mathrm{X} 1$ & -4.359 & & $\mathrm{Y} 1-\mathrm{Y} 1$ & -9.86 \\
\hline $\mathrm{X} 2-\mathrm{X} 2$ & -2.2095 & & $\mathrm{Y} 2-\mathrm{Y} 2$ & -5.22 \\
\hline $\mathrm{X} 3-\mathrm{X} 3$ & -0.082 & & $\mathrm{Y} 3-\mathrm{Y} 3$ & -7.36 \\
\hline $\mathrm{X} 4-\mathrm{X} 4$ & 6.656 & & $\mathrm{Y} 4-\mathrm{Y} 4$ & -9.51 \\
\hline$\Sigma$ & $\mathbf{0 . 0 0 5 5}$ & & $\Sigma$ & $\mathbf{- 3 2 . 0}$ \\
\hline
\end{tabular}

Tabla 9. Coordenadas de diseño de aplicación de fuerzas sísmicas generadas por los Procedimientos I y II (PI y PII respectivamente).

\begin{tabular}{|c|c|c|}
\hline NIVEL & $\mathbf{X}_{\mathbf{d P I}}(\mathbf{m})$ & $\mathbf{X}_{\mathbf{d P I I}}(\mathbf{m})$ \\
\hline 3 & 10.015 & 10.014 \\
\hline 2 & 10.096 & 10.095 \\
\hline 1 & 11.378 & 11.383 \\
\hline
\end{tabular}

En la tabla $9 X_{d}$ es la coordenada en donde finalmente se aplicarán las fuerzas laterales, mientras que PI y PII son los procedimientos que calculan la excentricidad estática en el entrepiso y en el nivel, respectivamente. De ésta tabla se observa que las coordenadas de aplicación de las cargas laterales calculadas por ambos procedimientos son iguales, lo cual permite concluir, que es indistinto obtener las excentricidades de diseño en los entrepisos o en los niveles. Es pertinente aclarar que la determinación de las coordenadas finales de aplicación de cargas se obtiene con mayor facilidad utilizando únicamente los centros de rigidez de nivel. En la tabla 10 se comparan los cortantes basales de los marcos del edificio estudiado obtenidos con los métodos descritos.

Tabla 10. Comparación de cortantes basales de los marcos para la dirección de análisis considerada. Método de Goel (Goel) vs. Procedimiento I (PI).

\begin{tabular}{|c|c|c|}
\hline MARCO & Goel (ton) & PI (ton) \\
\hline $\mathbf{X 1 - X 1}$ & -4.359 & -4.359 \\
\hline $\mathbf{X 2 - X 2}$ & -2.2095 & -2.21 \\
\hline $\mathbf{X 3 - X 3}$ & -0.082 & -0.084 \\
\hline $\mathbf{X 4 - X 4}$ & 6.656 & 6.711 \\
\hline$\Sigma$ & $\mathbf{0 . 0 0 5 5}$ & $\mathbf{0 . 0 5 8}$ \\
\hline
\end{tabular}

\begin{tabular}{|c|c|c|}
\hline MARCO & Goel (ton) & PI (ton) \\
\hline Y1-Y1 & -9.86 & -9.85 \\
\hline Y2-Y2 & -5.22 & -5.221 \\
\hline Y3-Y3 & -7.36 & -7.359 \\
\hline Y4-Y4 & -9.51 & -9.5 \\
\hline$\Sigma$ & $\mathbf{- 3 2}$ & $\mathbf{- 3 1 . 9 3}$ \\
\hline
\end{tabular}

Es claro que, para fines prácticos, los resultados obtenidos por medio de ambos procedimientos son iguales.

\section{COMENTARIOS AL MÉTODO SÍSMICO ESTÁTICO}

Tradicionalmente ha existido controversia ante la posibilidad de utilizar el Método Estático (ME) o el Método Modal Espectral (MME) para determinar las fuerzas de diseño de una estructura debido principalmente a las diferencias metodológicas que ambos presentan al momento de su aplicación. En las líneas siguientes se discuten las ventajas y desventajas de aplicar uno u otro método, incluyéndose una breve discusión sobre una forma alternativa de considerar los efectos de la excentricidad accidental en los MME. 
El ME ha sido utilizado cotidianamente debido a la relativa facilidad con la que pueden determinarse las fuerzas de diseño independientemente de que para aplicarlo sea necesaria la determinación de los centros de rigidez. En particular para el Distrito Federal las normas vigentes (NTCDS, 1995) y las normas propuestas (NTCDS, 2001) tienen como requisito establecer la combinación de cargas más desfavorable para cada marco o muro, lo cual tiene un alto costo hora - hombre puesto que la determinación de tales combinaciones no se encuentra implementada en la mayoría de los programas comerciales de análisis estructural disponibles en México. Para la aplicación estricta del ME según las (NTCDS, 1995) es obligatorio el cálculo de las excentricidades estructurales de entrepiso, pues se requiere de la comparación entre cada una de ellas en la dirección correspondiente para cumplir con el requisito de considerar, como mínimo, la mitad del máximo valor calculado de excentricidad estructural para los entrepisos que se hallan abajo del que se considera; este requisito también está considerado en la propuesta (NTCDS, 2001). Por último y con el fin de clasificar a la estructura como regular, irregular o fuertemente irregular, las NTCDS vigentes y las propuestas requieren la comparación de la excentricidad estática con un porcentaje de una de las dimensiones en planta del entrepiso correspondiente.

En lo que respecta a los factores de amplificación que afectan a la excentricidad estática $(\gamma)$ estos se derivan de estudios de modelos simplificados de un nivel y tres grados de libertad que se suponía representaban adecuadamente a los edificios reales, obteniéndose los primeros usando las rigideces desacopladas de un edificio de varios niveles sin tomar en cuenta el acoplamiento entre los modos, extrapolando los resultados de estos modelos en edificios reales con fines reglamentarios sin verificar la validez de tal consideración. Los factores de amplificación dinámica obtenidos de esta manera se aplican en toda la altura del edificio como si fuese constante lo cual dista mucho de la realidad y no se repara en las características particulares de cada estructura.

Por otro lado a diferencia del ME, la mayoría de los programas de análisis estructural disponibles en México tienen implementado el MME permitiendo seleccionar una regla de combinación de un conjunto de ellas. De acuerdo con la reglamentación mexicana el MME puede utilizarse para el análisis de toda estructura, teniendo como ventaja la estimación de su respuesta máxima a partir de un espectro de diseño, sin necesidad de buscar la combinación más desfavorable para cada marco o muro considerando tantos modos de vibrar como se juzgue necesario. La aplicación del método no esta limitada a estructuras con una cierta clasificación de regularidad, aspecto que se debe revisar previamente cuando se desea usar el ME.

Adicionalmente, es de esperarse que la calidad de los resultados obtenidos a partir de un análisis del tipo modal espectral sea superior a los derivados de análisis estáticos, puesto que los primeros tienen una fundamentación teórica más sólida basada en teoría de probabilidades permitiendo incluir el efecto de acoplamiento entre los modos, aspectos que el ME por ser simplista no contempla. En lo que se refiere a la amplificación dinámica, el MME considera las características particulares de las estructuras ya que tienen implícito este efecto y no requiere de la aplicación de factores estandarizados que por su naturaleza no corresponden a la estructura analizada. Por las razones expuestas, se considera razonable adoptar para el Reglamento el MME como el procedimiento para el análisis de edificios (independientemente de sus condiciones de regularidad), dejando como segunda opción el uso del procedimiento estático usando 
excentricidades de nivel con la ventaja sobre las excentricidades de entrepiso de no requerir el cálculo explicito de los centros de torsión.

En relación con esta sugerencia, es importante mencionar que uno de los aspectos de los que adolece el MME es que, hasta la fecha, las diferentes ediciones de las NTCDS no mencionan con claridad como se debe considerar la excentricidad accidental en la aplicación del MME. La propuesta de las (NTCDS, 2001) es la primera que recomienda un procedimiento análogo al que se hace en el ME al proponer que las fuerzas sísmicas resultantes en cada dirección de análisis se trasladen transversalmente $\pm 0.1 \mathrm{~b}$ aplicando el mismo signo en todos los niveles. En este trabajo se sugiere como procedimiento alterno para tomar en cuenta el efecto de torsión accidental en el MME, aplicar un momento estático concentrado del mismo signo en todos los niveles igual a $\pm 0.1 \mathrm{bF}$ yj, ya que es posible demostrar la igualdad entre lo planteado en la propuesta (NTCDS, 2001) y la aplicación del momento concentrado apelando a lo desarrollado en este texto. Sin embargo, se considera importante cuestionar la naturaleza de la magnitud de la excentricidad accidental, pues así como los factores de amplificación dinámica, este parámetro se ha derivado de estudios de modelos de un nivel y tres grados de libertad, sin verificar su validez de forma experimental, ya que esta excentricidad (y en consecuencia el momento derivado de ella), pretende considerar los efectos que sobre la estructura tienen factores que están fuera del alcance de un modelo numérico y que son de diversa índole, aplicándose de igual manera en cualquier procedimiento de análisis que se adopte.

\section{CONCLUSIONES Y RECOMENDACIONES}

La discusión presentada sobre la aplicación del ME para el análisis sísmico de estructuras asimétricas permitió aclarar la incertidumbre sobre dos procedimientos comúnmente empleados, demostrándose que es posible obtener los mismos resultados utilizando un método más simplificado (método de Goel). Cabe destacar que en tanto continúe vigente la aplicación del Método Sísmico Estático varias de las conclusiones a las que se llegaron permitirán modificar en el futuro las Normas Técnicas Complementarias para Diseño por Sismo así como los requisitos para su aplicación.

En este artículo se demuestra que en la aplicación del ME el emplear excentricidades de nivel o de entrepiso conduce a los mismos resultados debido a que son equivalentes, obteniéndose los resultados deseados de forma más simple al considerar las primeras, sin embargo, en cualquiera de las versiones presentadas del ME no se evita el cálculo de las combinaciones de carga más críticas para diseño.

Se encuentra que al comparar el ME con el Método Modal Espectral (MME) desde el punto de vista de fundamentación teórica y facilidad de aplicación, el ME queda en desventaja ante el MME puesto que el último se encuadra dentro de un marco teórico y requiere de menos combinaciones que el ME. Adicionalmente, el MME es de aplicación general y no requiere del cálculo de ningún tipo de excentricidad ni de la clasificación de la estructura por analizar como ocurre en la aplicación estricta del ME según el (RCDF, 1993), donde es obligatorio el cálculo de las excentricidades estáticas de entrepiso, concluyéndose que debido a las bases teóricas del 
MME y dadas las herramientas de análisis disponibles el ME es obsoleto. De seguirse permitiendo el uso del ME sería recomendable que se requiriera la determinación de la excentricidad de nivel en vez de la de entrepiso pues esto facilitaría su aplicación y la clasificación de las estructuras en términos de regularidad. Es importante resaltar que la excentricidad estática a la que se refieren las NTCDS es la excentricidad de entrepiso, cuya magnitud es diferente de la excentricidad de nivel, en consecuencia no se deberá utilizar esta última para efectos de clasificación y criterio de selección del análisis de las estructuras siendo necesario redefinir los limites a los que hace mención la norma.

Para tomar en cuenta el efecto de torsión accidental en el MME, se sugiere como procedimiento alterno al descrito en la propuesta a las (NTCDS, 2001) aplicar un momento estático concentrado (igual a $\pm 0.1 \mathrm{bF}_{\mathrm{j}}$ ) considerando el mismo signo para todos los niveles, pues como se muestra en este trabajo la aplicación de un momento concentrado en cualquier punto de un diafragma rígido causa los mismos efectos.

\section{RECONOCIMIENTOS}

Se agradece a la Dirección General de Asuntos del Personal Académico el patrocinio del Proyecto IN112600, "Desarrollo de Criterios de Diseño Sísmico por Torsión" y a Alfonso Islas, Gilberto Miranda y Guillermo Roeder su apoyo en la implantación de los modelos.

\section{APÉNDICE I. DEMOSTRACIÓN DEL MÉTODO DE GOEL POR EL PRINCIPIO DE SUPERPOSICIÓN}

Por claridad se presenta la demostración del Método de Goel usando el principio de superposición. Como se mencionó, este método considera la excentricidad estática de nivel por medio de la cual se derivan las excentricidades de diseño, quedando definidas las coordenadas de aplicación de la fuerza lateral por las ecs. A1 y A2:

$e_{d j}=1.5 e_{s j}+0.1 b$

$e_{d j}=e_{s j}-0.1 b$

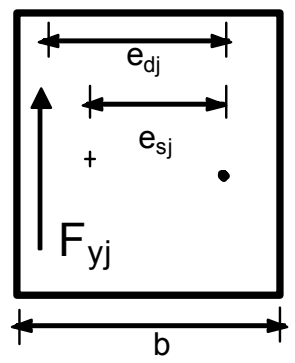

Figura A1. Condición de carga para la excentricidad de diseño en el $j$-ésimo nivel. 
Habiendo calculado $\mathrm{e}_{\mathrm{dj}}$ la carga lateral queda situada en el $j$-ésimo nivel como se muestra en la fig. A1.

La condición de carga mostrada en la fig. A1 puede descomponerse a su vez en:

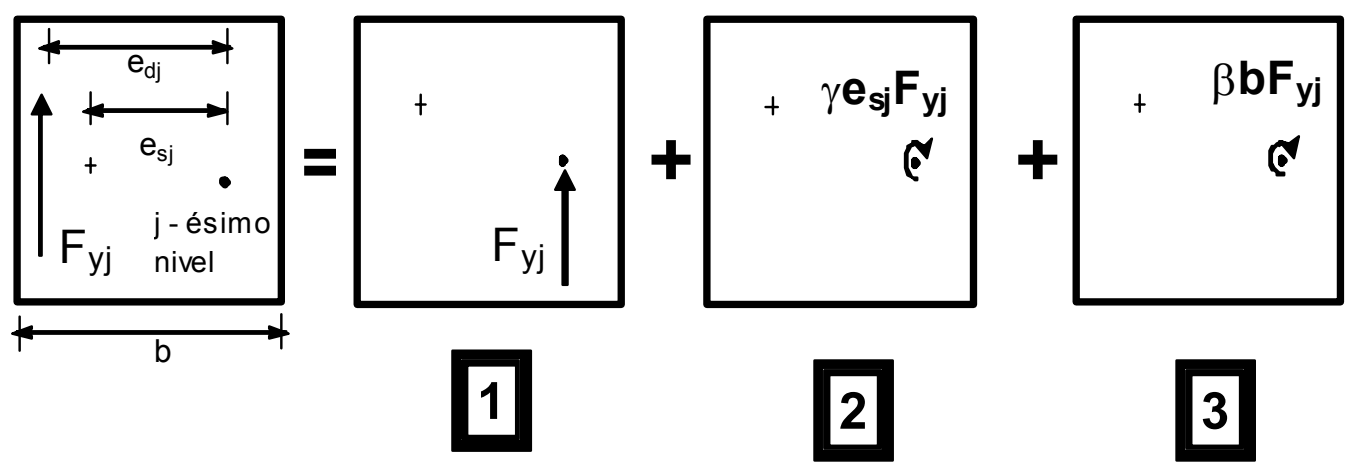

Figura A2. Descomposición del estado de carga original presentado en la fig. A1.

En la condición de análisis 1, fig. A2, al estar aplicadas las cargas en los centros de rigidez de nivel solo ocurre traslación en el sentido de éstas, por tanto, esta condición es equivalente a la mostrada en la fig. A3.
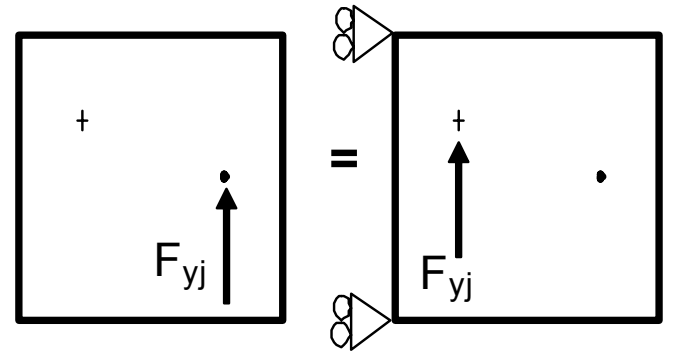

Figura A3. Condición de carga que produce traslación en el j-ésimo nivel, condición 1.

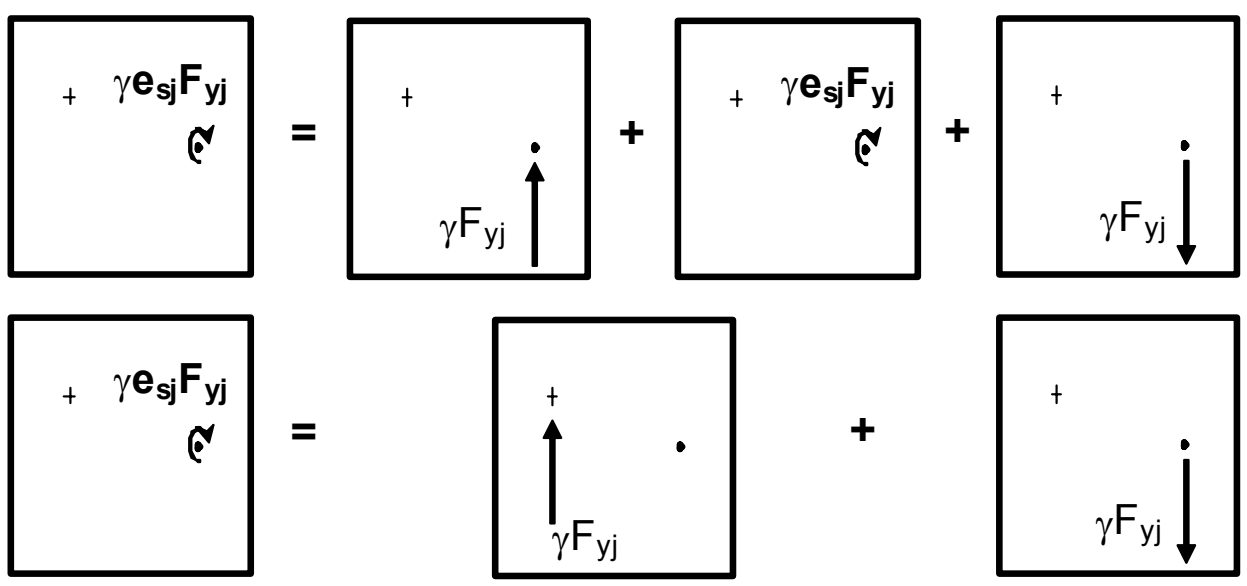

Figura A4. Descomposición del estado de análisis 2, de la fig. A2 
La condición de análisis 2 de la fig. A2, puede expresarse como se ilustra en la fig. A4.
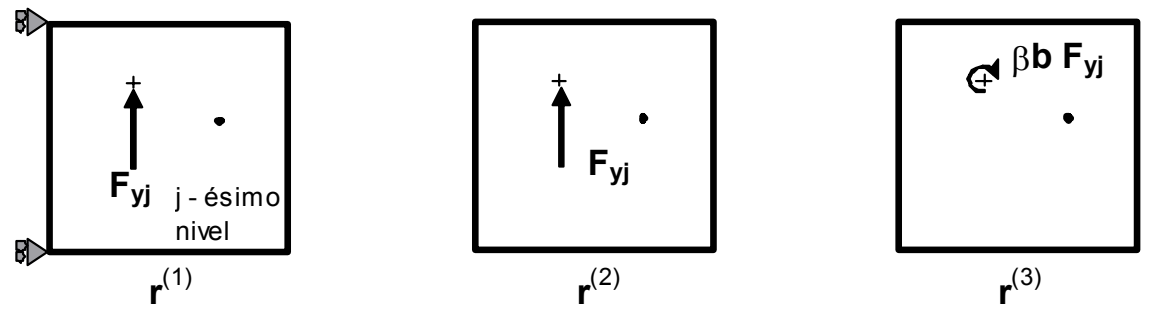

Figura A5. Condiciones de análisis $\mathrm{r}^{(1)}, \mathrm{r}^{(2)}, \mathrm{r}^{(3)}$

Para la condición de análisis 3 , la aplicación de un momento concentrado en cualquier punto de un diafragma rígido conduce a los mismos resultados, para los fines del estudio dicho momento debido a la excentricidad accidental se considerará aplicado en el centro de masas. Si llamamos $r^{(1)}, r^{(2)}, r^{(3)}$ a los análisis de la fig. A5:

La condición inicial se obtiene al combinar linealmente las respuestas de $r^{(1)}, r^{(2)} \mathrm{y} \mathrm{r}^{(3)} \mathrm{de}$ acuerdo con la siguiente regla:

$r=r^{(1)}+\gamma\left(r^{(2)}-r^{(1)}\right) \pm r^{(3)}$

Que es la expresión para el cálculo de la respuesta de la estructura en el método de Goel y Chopra con los correspondientes valores de $\gamma$ y signo de $r^{(3)}$.

\section{REFERENCIAS}

Bazán, E (1978), “Análisis sísmico de edificios con muros rigidizantes”, IMCYC, Vol. 41, No. 91, México, D. F., pp. 17-31.

Cheung, V W y W K Tso (1986a), "Decoupling of equations of equilibrium in lateral load analysis of multistory buildings", Computers and Structures, Vol. 23, No. 5, pp. 679-684.

Cheung, V W T y W K Tso (1986b), "Eccentricity in irregular multistory buildings", Canadian Journal of Civil Engineering, Vol. 13, No. 1, pp. 46-52.

Chipol, A (2001), "Estudio de la respuesta sísmica de modelos tridimensionales de edificios torsionalmente acoplados", Tesis de Maestría. División de Estudios de Posgrado, Facultad de Ingeniería, Universidad Nacional Autónoma de México, México.

Damy, J y S Alcocer (1987), "Obtención del centro de torsión de edificios", Memorias del VII Congreso Nacional de Ingeniería Sísmica, SMIS, Querétaro, México, pp. C-60 a C-67.

Goel, R K y A K Chopra (1993), "Seismic code analysis of buildings without locating centers of rigidity", ASCE Journal of Structural Engineering, Vol. 119, No. 10, pp. 3039-3055.

Goel, R K y A K Chopra (1995), "Seismic code analysis of buildings without locating centers of rigidity", (cierre por los autores), ASCE Journal of Structural Engineering, Vol. 121, No. 4, pp. 793-794. 
Humar, J L (1984), "Design for seismic torsional forces", Canadian Journal of Civil Engineering, Vol. 11, No. 2, junio, pp.150-163.

Newmark, N M y E Rosenblueth (1975), Fundamentals of earthquake engineering, Prentice Hall, Inc. Englewood Cliffs, N.J.

NTCDS (1995), "Normas Técnicas Complementarias para Diseño por Sismo", Gaceta Oficial del Departamento del Distrito Federal, México.

NTCDS (2001), Propuesta de modificación a las "Normas Técnicas Complementarias para Diseño por Sismo", Sociedad Mexicana de Ingeniería Estructural, abril, México.

Poole, R A (1977), “Analysis for torsion employing provisions of NZRS4203: 1974” Bulletin of the New Zealand National Society for Earthquake Engineering, Vol. 10, No. 4, pp. 219-225.

RCDF (1993), Reglamento de Construcciones del Distrito Federal, Diario Oficial de la Federación, México.

UBC (1991), "Uniform Building Code-Earthquake Regulations", International Conference of Building Officials, Whittier, CA.

Zárate G (2002), "Evaluación de enfoques para análisis y diseño sísmico de edifícios asimétricos". Tesis de Maestría, Programa de Posgrado en Ingeniería. Facultad de Ingeniería, Universidad Nacional Autónoma de México, México. 